\title{
Caracterización de la composición y estructura florística en las geoformas asociadas a una vega, en el Centro Regional de Investigación - INIA Kampenaike, en la región de Magallanes, Chile
}

\author{
Characterization of the composition and floristic structure \\ in the geoforms associated a wet meadow, \\ INIA Kampenaike Research Center, Magallanes region, Chile \\ Erwin Domínguez Díaz ${ }^{1} \&$ Jorge Ivelic-Sáez ${ }^{1}$
}

\section{Resumen}

Un estudio sobre la estructura y composición florística se realizó en tres geoformas asociadas a una vega (humedal) en el Centro Regional de Investigación INIA Kampenaike, ubicado en la zona intermedia de la estepa magallánica (5242’27”S, 7054’52”O). La hipótesis de trabajo fue que el pastoreo sobre geoformas asociadas a una vega genera diferencias en la composición y estructura florística de la vegetación. En este contexto, los objetivos específicos fueron: 1) describir los componentes taxonómicos, 2) caracterizar la biodiversidad florística y 3) evaluar la similitud o diferencias de la vegetación, junto con determinar la contribución de las especies que estructuran las comunidades de plantas en geoformas asociadas a una vega. El análisis fue mediante el uso de 57 cuadrantes de $1 \mathrm{~m}^{2}$. Se identificaron a nivel específico 55 taxones, 1 de la División Ascomycota, 2 de la División Bryophyta y 52 de la División Tracheophyta (plantas superiores), 19 especies de la Clase Liliopsida y 33 especies de la Clase Magnoliopsida, distribuidas en 23 familias, 41 géneros, 53 especies y dos especímenes que no pudieron ser identificados. Los índices de biodiversidad más altos corresponden a las geoformas de Ladera Sur y Norte respectivamente, en comparación a la geoforma Depresión (Vega), donde se puede apreciar una presión antropogénica debido a la intensidad del pastoreo, la que ha causado una disminución en la biodiversidad en términos de número de especies y cobertura, lo cual se ajusta a otros estudios realizados en vegas en la
Patagonia. Para evaluar estructura del ensamble de las comunidades entre las geoformas, se realizó un análisis de ordenamiento multidimensional (nMDS) y se determinó la significancia de los grupos mediante el método ANOSIM $(\mathrm{R}=$ 0,6902; $p \leq 0,0001)$. El análisis SIMPER mostró que las plantas que contribuyeron de manera más significativa fueron en la geoforma Depresión que corresponde a la vega. Ellas son: Agrostis meyenii, Taraxacum officinale y Hordeum pubiflorum con más del $50 \%$ de la cobertura promedio. En la geoforma Ladera Sur existió una mayor heterogeneidad taxonómica, aunque sólo una especie (Poa spiciformis) generó el valor más alto de la similitud dentro de este grupo con una cobertura promedio de $13 \%$. En cambio, en la geoforma Ladera Norte, las especies que más contribuyeron a la similitud de este grupo fueron: Taraxacum officinale, Acaena pinnatifida y Aira caryophyllea con una cobertura promedio de $42 \%$. Estos resultados permiten cuantificar que el pastoreo excesivo genera efectos negativos que se manifiestan en cambios en la vegetación, debido a la sustitución de especies por otros taxones que son propios de sectores áridos.

\section{Palabras clave:}

geoformas, vegas, diversidad florística, pastoreo, Patagonia.

\footnotetext{
Instituto de Investigaciones Agropecuarias (INIA).

Casilla 277, Punta Arenas, Chile.

\edominguez@inia.cl
} 


\section{Abstract}

A study on the structure and floristic composition was carried out in three geoforms associated with a wet meadow (wetland) at the INIA Kampenaike Regional Research Center, located in the intermediate zone of the Magellanic steppe (52.42'27"S, $\left.70^{\circ} 54^{\prime} 52^{\prime \prime} \mathrm{W}\right)$. The working hypothesis was that grazing on geoforms associated with a wet meadow generates differences in the composition and floristic structure of the vegetation. In this context, the specific objectives were: 1) describe the taxonomic components, 2) characterize the floristic biodiversity and 3) evaluate the similarity or differences of the vegetation, together with determining the contribution of the species that structure the plant communities in geoforms associated with a wet meadow. The analysis was through the use of 57 quadrants of $1 \mathrm{~m}^{2}$. At a specific level, 55 taxa were identified, 1 from the Ascomycota Division, 2 from the Bryophyta Division and 52 from the Tracheophyta Division (higher plants), 19 species of the Liliopsida Class and 33 species of the Magnoliopsida Class, distributed in 23 families, 41 genera, 53 species and two specimens that could not be identified. The highest biodiversity indices correspond to the geoforms of the South and North slopes respectively, compared to the Depression (wet meadow) geoform, where anthropogenic pressure can be seen due to the intensity of grazing, which has caused a decrease in biodiversity in terms of number of species and coverage, which is in line with other studies carried out in wetlands in Patagonia. To assess the structure of the assemblage of the communities between the geoforms, a multidimensional ordering analysis (nMDS) was performed and the significance of the groups was determined using the ANOSIM method $(\mathrm{R}=0.6902 ; \mathrm{p} \leq 0.0001)$. The SIMPER analysis showed that the plants that contributed most significantly were in the Depression geoform that corresponds to the wet meadow. They are: Agrostis meyenii, Taraxacum officinale and Hordeum pubiflorum with more than $50 \%$ of the average coverage. In the South Slope geoform there was a greater taxonomic heterogeneity, although only one species (Poa spiciformis) generated the highest value of similarity within this group with an average coverage of $13 \%$. In contrast, in the North Slope geoform, the species that most contributed to the similarity of this group were: Taraxacum officinale, Acaena pinnatifida and Aira caryophyllea with an average coverage of $42 \%$. These results allow quantifying that excessive grazing generates negative effects that are manifested in changes in vegetation, by the substitution of species, by other taxa that are typical of arid sectors.

\section{Key words:}

geoforms, wet meadow,

floristic diversity, grazing, Patagonia.

\section{INTRODUCCIÓN}

Las vegas o mallines en la Patagonia son un tipo de humedal y se caracterizan por ser áreas saturadas de agua, en forma permanente o temporal durante el año, siendo éste el factor dominante que determina la naturaleza del desarrollo de un suelo abundante en materia orgánica y de una vegetación herbácea típica, formada por juncáceas, ciperáceas, gramíneas (Pisano, 1977). Particularmente, las vegas o mallines, constituyen un tipo de humedal que es frecuente, pero no abundante en las extensas planicies en la estepa patagónica en la región de Magallanes, la que puede cubrir una superficie aproximada de 200.000 hectáreas (SAG, 2004). Estos humedales se desarrollan comúnmente en depresiones entre morrenas o fondos de valles. En la estepa su formación requiere del aporte de agua subterránea o estacional, generada por el escurrimiento superficial de los deshielos o de las precipitaciones estacionales. Las vegas constituyen sistemas de alto valor productivo por ser una fuente de agua y alimento para la ganadería (bovina y ovina en especial), principalmente por el aporte de forraje de calidad, con alto valor nutricional (Buono et al. 2010).

Sus principales funciones $y$ servicios ecosistémicos son: 1) la regulación hídrica manteniendo el agua excedente de la estación húmeda, 2) regulación de los caudales de chorrillos o arroyos, mitigando las crecidas y 3) actúan como filtro y retención de sedimentos. Además, por ser un humedal, tiene un rol importante en el secuestro de carbono debido al alto contenido de materia orgánica de sus suelos (Houghton, 2007). Las causas más frecuentes que conducen a la degradación de estos ambientes son el 
sobrepastoreo (Utrilla et al. 2005) y la intervención intencional de la red de drenaje (Lord et al. 2011; Scopel et al. 2017). Este último proceso provoca el descenso del nivel freático, causando cambios en la vegetación (Soriano \& Movia, 1986; Vázquez et al. 2013). Por otra parte, las características de las geoformas asociadas a las vegas son importantes, por la composición florística que éstas tienen, cuando se piensa en su manejo o su restauración (Olff \& Ritchie, 1998). Los estudios sobre vegas en Patagonia, en su mayoría, se han focalizado en los aspectos edáficos, sedimentológicos, topográficos, hídricos, mapeos y procesamiento de imágenes satelitales (Mazzoni \& Rabassa, 2013; Vázquez et al. 2013; Gaitán et al. 2015; Grima et al. 2015); otros se refieren a la distribución espacial de las comunidades vegetales (Gandullo \& Schmid, 2001; Gaitán et al. 2011; Mazzoni \& Rabassa, 2013; Vázquez et al. 2013).

Por otra parte, las vegas ocupan el $22 \%$ de la superficie total en el Centro Regional de Investigación INIA Kampenaike $y$ han estado sujetas al pastoreo durante 44 años, tanto por animales domésticos, como bovinos y ovinos, soportando un régimen de ganadería de tipo rotativo, semi-intensivo, con cargas moderadas. Las respuestas de estas comunidades vegetales y sus geoformas asociadas, frente a la acción de los herbívoros domésticos, no han sido estudiadas. La hipótesis de este trabajo es que el pastoreo sobre las geoformas asociadas a una vega genera diferencias en la composición y estructura florística de la vegetación. En este contexto, los objetivos de este estudio son: 1) describir los componentes taxonómicos, 2) caracterizar la biodiversidad florística y 3) evaluar la similitud o diferencias de la vegetación, junto con determinar la contribución de las especies que estructuran las comunidades de plantas en geoformas asociadas a una vega.

\section{MATERIAL Y MÉTODOS}

\section{Sitio de estudio}

El Centro Regional de Investigación INIA Kampenaike está ubicado a $60 \mathrm{~km}$ al NE de la ciudad de Punta Arenas (5242'27”S, 7054'52”O) (Fig. $1)$, en la zona intermedia de la estepa magallánica y abarca una superficie aproximada de 10.070 ha. La precipitación media anual es de $300 \mathrm{~mm}$ distribuidos de manera relativamente uniforme a lo largo del año. La temperatura promedio anual es de $8,5^{\circ} \mathrm{C}$, la máxima del mes cálido es de $24^{\circ} \mathrm{C}$ y la mínima del mes más frío es de $-7^{\circ} \mathrm{C}$ (https://agrometeorologia.cl/). El paisaje incluye geoformas de origen glacifluvial que se manifiestan en cerros bajos con ondulaciones y depresiones. Las vegas cubren el 22\% (2.259 ha) de la superficie total. El 20 de agosto de 2019, se realizó un reconocimiento geomorfológico en el potrero Vega 1, mediante el empleo de un vehículo aéreo no tripulado (VANT) Phantom 4 Pro 2v, identificando tres geoformas (Ladera Sur, Depresión y Ladera Norte). Históricamente, el potrero Vega 1 (de 9 ha) ha sido usado para el encaste bovino de 25 a 27 vacas con un toro, desde enero a febrero, descansando el campo por tres meses. Luego, en mayo ingresan 400 a 600 ovejas, para encaste, por 35 días, quedando libre de animales hasta el mes de enero del año siguiente, cumpliendo un ciclo de rotación.

\section{Diseño de muestreo}

El 20 de noviembre de 2019, se realizó un muestreo de la vegetación, utilizando 57 cuadrantes de $1 \mathrm{~m}^{2}$ ( $\left.2 \times 0,5 \mathrm{~m}\right)$ distribuidos cada $10 \mathrm{~m}$, en tres transectos de $190 \mathrm{~m}$ de largo, orientados de sur a norte. En cada parcela se estimó la riqueza de especies y la cobertura vegetal (\%). Los trabajos de identificación de plantas se realizaron con claves de acuerdo a la Flora Patagónica de INTA (Correa, 1969, 1971, 1978, 1984, 1985, 1988, 1999). La nomenclatura de las especies, su hábito y origen, se basó en el Catálogo de Plantas Vasculares de Chile (Rodríguez \& Marticorena, 2019).

\section{Análisis estadístico}

La complejidad de la comunidad vegetal presente en cada unidad muestral se estimó a través del índice de dominancia (D), índice de Simpson (1-D) e indice de diversidad ShannonWiener ( $\left.\mathrm{H}^{\prime}\right)$. Con los datos obtenidos se realizó la prueba de Kruskal-Wallis con la corrección de Bonferroni, para detectar diferencias entre las geoformas, debido a que los datos no mostraron distribución normal (prueba de Shapiro-Wilk). La 


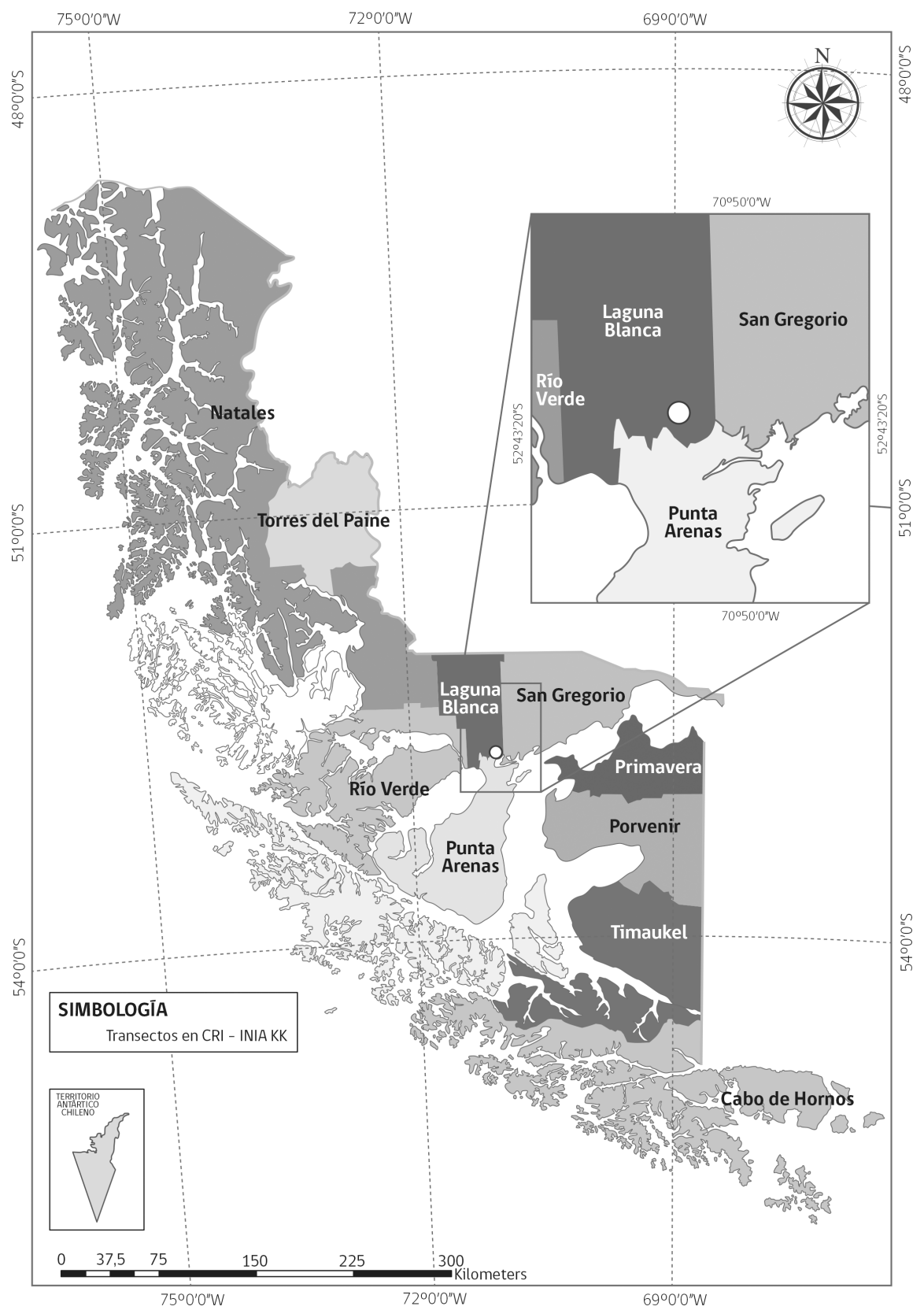

Fig. 1. Ubicación de los transectos botánicos para las tres geoformas asociadas a una vega. Estas son: Ladera Norte, Depresión y Ladera Sur, ubicadas en el CRI - INIA Kampenaike

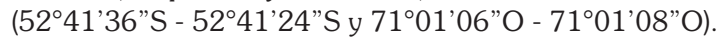

estructuración del ensamble de las comunidades se representa y contrasta gráficamente utilizando un ordenamiento de escalamiento multidimensional no paramétrico (nMDS) (Kruskal, 1964), sobre una matriz de distancia de disimilaridad de Bray-Curtis (Bray \& Curtis, 1957; Clarke et al. 2006; Härdle,
\& Simar, 2012).), la cual utiliza la información de composición de especies con su respectiva cobertura. Luego, se comparó la composición de especies entre las geoformas con ANOSIM (Clarke \& Green, 1988), utilizando 9.999 permutaciones con el objeto de estimar un nivel de significancia 
$p<0,05$ entre las geoformas; bajo la hipótesis nula de que no hay diferencias en la composición florística entre ellas, producto del pastoreo. Finalmente, para determinar la contribución de las especies en la estructuración de la comunidad de plantas, se realizó un análisis de porcentaje de similitud (SIMPER) (Clarke, 1993), de modo de indicar cuantitativamente cuáles son las especies que explican las diferencias entre grupos. Todos los análisis, tanto univariados como multivariados, se realizaron en el programa PAST (Palaeontological Statistics) v4.0 (Hammer et al. 2001).

\section{RESULTADOS}

En total se registraron 55 especies distribuidas en 23 familias y 41 géneros, de las cuales 42 especies son nativas y 11 no nativas. Se determinaron 52 taxones que corresponden a la División Tracheophyta (plantas superiores), 1 de la División Ascomycota y 2 de la División Bryophyta. La Clase mejor representada fue Magnoliopsida con 33 especies, seguida por 19 especies de la Clase Liliopsida. Dos especímenes no pudieron ser identificados a nivel específico, por no presentar estructura reproductiva. Las familias mejor representadas fueron: Poaceae con 12 especies y Asteraceae con 8 (Tabla 1). En todas las geoformas se encontraron especies introducidas: 6 especies en la Ladera Sur y Norte, y 8 especies en la Depresión (Tablas 1 y 4). Por otra parte, los resultados de los parámetros comunitarios que corresponden a la geoforma Depresión, indican que difiere significativamente en la riqueza de especies (S) respecto a las geoformas Ladera Sur y Norte; para la cobertura vegetal (\%), la Ladera Sur difiere significativamente de la geoforma Depresión, no así la Ladera Norte para el índice de Shannon (H'), la geoforma Depresión indica una menor diversidad, la cual difiere significativamente de las otras dos geoformas (Tabla 2).

El análisis de ordenamiento multidimensional (nMDS), basado en las coberturas de especies, para evaluar la similitud o diferencias de la vegetación relacionada a las tres geoformas asociadas a la vega, mostró evidencias de agrupaciones genuinas de las muestras involucradas (geoformas), las que se pueden apreciar en la Fig. 2. Los resultados obtenidos con el ANOSIM sumado al índice de Bray-Curtis proporcionaron diferencias significativas entre las tres geoformas estudiadas $(R=0,6902$;

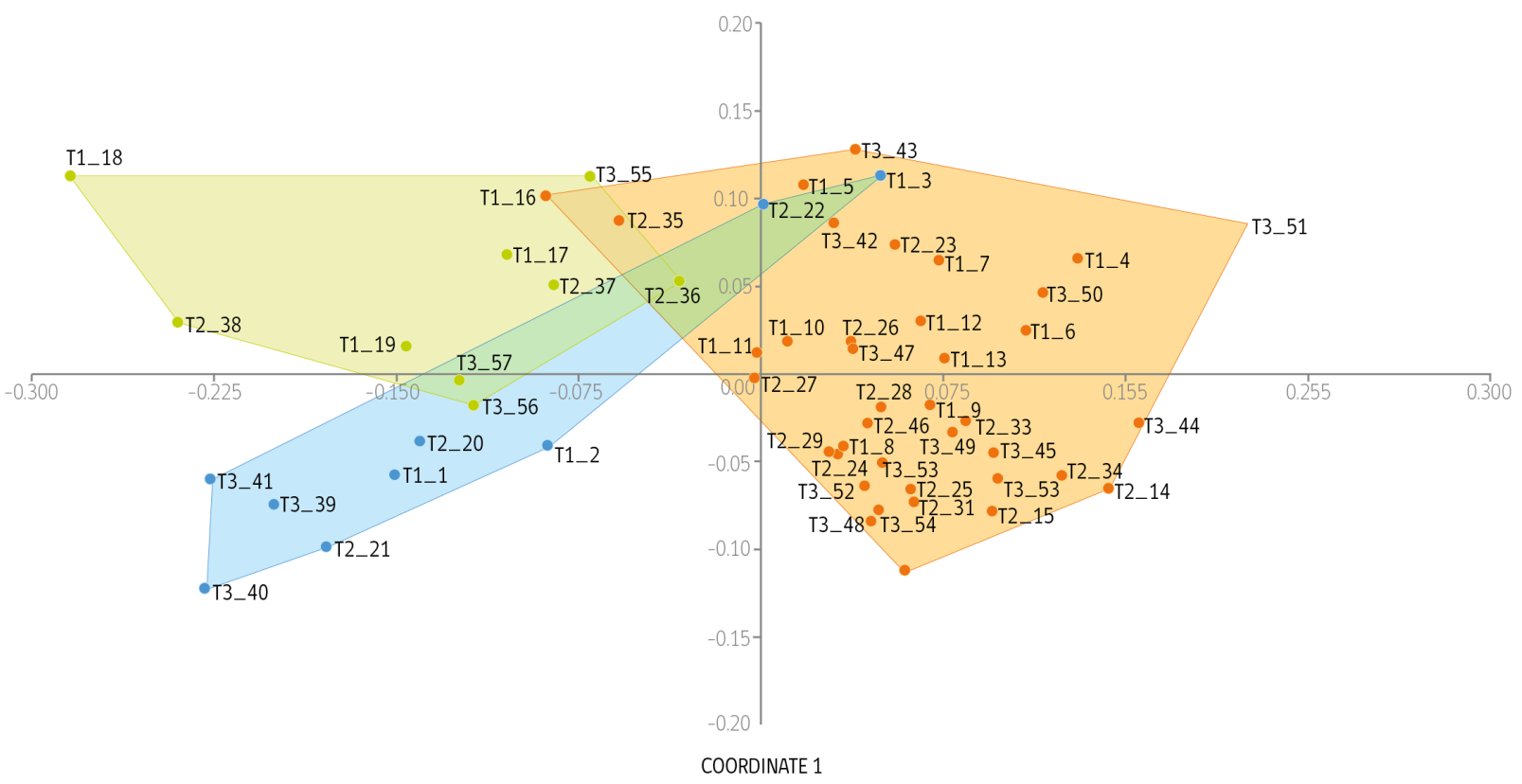

Fig. 2. Ordenamiento de escalamiento multidimensional no paramétrico (nMDS, estrés = 0,1855) que muestra agrupaciones entre las tres geoformas estudiadas. Celeste = Ladera Sur; Naranja = Depresión que corresponde a la vega y Verde = Ladera Norte. 
Tabla 1. Flora registrada en el área de estudio, ordenada según la clasificación de Rodríguez \& Marticorena (2019).

\begin{tabular}{|c|c|c|c|c|c|c|}
\hline ID & División & Clase & Familia & Especies & Hábito & Origen \\
\hline 1 & Tracheophyta & Liliopsida & Cyperaceae & Carex acaulis & Hierba, perenne & Nativa \\
\hline 2 & Tracheophyta & Liliopsida & Cyperaceae & Eleocharis nubigena & Hierba, perenne & Nativa \\
\hline 3 & Tracheophyta & Liliopsida & Cyperaceae & Carex sorianoi & Hierba, perenne & Nativa \\
\hline 4 & Tracheophyta & Liliopsida & Cyperaceae & $\begin{array}{l}\text { Carex } \\
\text { austroamericana }\end{array}$ & Hierba, perenne & Nativa \\
\hline 5 & Tracheophyta & Liliopsida & Iridaceae & Olsynium biflorum & Hierba, perenne & Nativa \\
\hline 6 & Tracheophyta & Liliopsida & Juncaceae & Luzula alopecurus & Hierba, perenne & Nativa \\
\hline 7 & Tracheophyta & Liliopsida & Juncaceae & $\begin{array}{l}\text { Juncus } \\
\text { scheuchzerioides }\end{array}$ & Hierba, perenne & Nativa \\
\hline 8 & Tracheophyta & Liliopsida & Poaceae & Agrostis meyenii & Hierba, perenne & Nativa \\
\hline 9 & Tracheophyta & Liliopsida & Poaceae & Poa spiciformis & Hierba, perenne & Nativa \\
\hline 10 & Tracheophyta & Liliopsida & Poaceae & Hordeum pubiflorum & Hierba, perenne & Nativa \\
\hline 11 & Tracheophyta & Liliopsida & Poaceae & Aira caryophyllea & Hierba, anual & Introducida \\
\hline 12 & Tracheophyta & Liliopsida & Poaceae & Poa pratensis & Hierba, perenne & Introducida \\
\hline 13 & Tracheophyta & Liliopsida & Poaceae & Festuca gracillima & Hierba, perenne & Nativa \\
\hline 14 & Tracheophyta & Liliopsida & Poaceae & Festuca magellanica & Hierba, perenne & Nativa \\
\hline 15 & Tracheophyta & Liliopsida & Poaceae & Phleum alpinum & Hierba, perenne & Nativa \\
\hline 16 & Tracheophyta & Liliopsida & Poaceae & Indet. & Indet. & Indet. \\
\hline 17 & Tracheophyta & Liliopsida & Poaceae & $\begin{array}{l}\text { Rytidosperma } \\
\text { virescens }\end{array}$ & Hierba, perenne & Nativa \\
\hline 18 & Tracheophyta & Liliopsida & Poaceae & Festuca pyrogea & Hierba, perenne & Nativa \\
\hline 19 & Tracheophyta & Liliopsida & Poaceae & $\begin{array}{l}\text { Alopecurus } \\
\text { magellanicus }\end{array}$ & Hierba, perenne & Nativa \\
\hline 20 & Tracheophyta & Magnoliopsida & Apiaceae & Azorella monantha & Subarbusto & Nativa \\
\hline 21 & Tracheophyta & Magnoliopsida & Asteraceae & Taraxacum officinale & Hierba, perenne & Introducida \\
\hline 22 & Tracheophyta & Magnoliopsida & Asteraceae & $\begin{array}{l}\text { Chiliotrichum } \\
\text { diffusum }\end{array}$ & Arbusto, perenne & Nativa \\
\hline 23 & Tracheophyta & Magnoliopsida & Asteraceae & Hypochaeris incana & Hierba, perenne & Nativa \\
\hline 24 & Tracheophyta & Magnoliopsida & Asteraceae & Hypochaeris palustris & Hierba, perenne & Nativa \\
\hline 25 & Tracheophyta & Magnoliopsida & Asteraceae & $\begin{array}{l}\text { Symphyotrichum } \\
\text { vahlii }\end{array}$ & Hierba, perenne & Nativa \\
\hline 26 & Tracheophyta & Magnoliopsida & Asteraceae & Senecio miser & Hierba, perenne & Nativa \\
\hline 27 & Tracheophyta & Magnoliopsida & Asteraceae & Leptinella scariosa & Hierba, perenne & Nativa \\
\hline 28 & Tracheophyta & Magnoliopsida & Asteraceae & Nassauvia aculeta & Hierba, perenne & Nativa \\
\hline 29 & Tracheophyta & Magnoliopsida & Berberidaceae & Berberis microphylla & Arbusto, perenne & Nativa \\
\hline 30 & Tracheophyta & Magnoliopsida & Berberidaceae & Berberis empetrifolia & Arbusto, perenne & Nativa \\
\hline 31 & Tracheophyta & Magnoliopsida & Boraginaceae & Myosotis stricta & $\begin{array}{l}\text { Hierba, anual o } \\
\text { perenne }\end{array}$ & Introducida \\
\hline 32 & Tracheophyta & Magnoliopsida & Brassicaeae & $\begin{array}{l}\text { Capsella bursa- } \\
\text { pastoris }\end{array}$ & $\begin{array}{l}\text { Hierba, anual o } \\
\text { bienal }\end{array}$ & Introducida \\
\hline 33 & Tracheophyta & Magnoliopsida & Brassicaeae & Draba verna & Hierba, anual & Introducida \\
\hline
\end{tabular}




\begin{tabular}{|c|c|c|c|c|c|c|}
\hline ID & División & Clase & Familia & Especies & Hábito & Origen \\
\hline 34 & Tracheophyta & Magnoliopsida & Calceolariaceae & Calceolaria uniflora & Hierba, perenne & Nativa \\
\hline 35 & Tracheophyta & Magnoliopsida & Campanulaceae & Lobelia oligophylla & Hierba, perenne & Nativa \\
\hline 36 & Tracheophyta & Magnoliopsida & Caryophyllaceae & Cerastium arvense & Hierba, perenne & Introducida \\
\hline 37 & Tracheophyta & Magnoliopsida & Caryophyllaceae & $\begin{array}{l}\text { Colobanthus } \\
\text { subulatus }\end{array}$ & Hierba, perenne & Nativa \\
\hline 38 & Tracheophyta & Magnoliopsida & Caryophyllaceae & Cerastium fontanum & Hierba, perenne & Introducida \\
\hline 39 & Tracheophyta & Magnoliopsida & Fabaceae & Adesmia lotoides & Hierba, perenne & Nativa \\
\hline 40 & Tracheophyta & Magnoliopsida & Gentianiaceae & $\begin{array}{l}\text { Gentianella } \\
\text { magellanica }\end{array}$ & Hierba, anual & Nativa \\
\hline 41 & Tracheophyta & Magnoliopsida & Geraniaceae & Erodium cicutarium & $\begin{array}{l}\text { Hierba, Anual o } \\
\text { bienal }\end{array}$ & Introducida \\
\hline 42 & Tracheophyta & Magnoliopsida & Geraniaceae & Geranium core-core & Hierba, perenne & Nativa \\
\hline 43 & Tracheophyta & Magnoliopsida & Oxalidaceae & Oxalis loricata & Hierba, perenne & Nativa \\
\hline 44 & Tracheophyta & Magnoliopsida & Plumbaginaceae & Armeria maritima & Hierba, perenne & Nativa \\
\hline 45 & Tracheophyta & Magnoliopsida & Polygonaceae & Rumex acetosella & Hierba, perenne & Introducida \\
\hline 46 & Tracheophyta & Magnoliopsida & Polygonaceae & Rumex crispus & Hierba, perenne & Introducida \\
\hline 47 & Tracheophyta & Magnoliopsida & Rosaceae & Acaena pinnatifida & Hierba, perenne & Nativa \\
\hline 48 & Tracheophyta & Magnoliopsida & Rosaceae & Acaena magellanica & Hierba, perenne & Nativa \\
\hline 49 & Tracheophyta & Magnoliopsida & Rosaceae & Acaena poeppigea & Hierba, perenne & Nativa \\
\hline 50 & Tracheophyta & Magnoliopsida & Schoepfiaceae & Arjona patagonica & Hierba, perenne & Nativa \\
\hline 51 & Tracheophyta & Magnoliopsida & Schoepfiaceae & Arjona pusilla & Hierba, perenne & Nativa \\
\hline 52 & Tracheophyta & Magnoliopsida & Violaceae & Viola maculata & Hierba, perenne & Nativa \\
\hline 53 & Bryophyta & Bryopsida & Pottiaceae & Syntrichia sp. & Briófita terrestre & Nativa \\
\hline 54 & Ascomycota & Lecanoromycetes & Peltigeraceae & Peltigera sp. & Epífita Liquen & Nativa \\
\hline 55 & Bryophyta & Bryopsida & Indet. & Briófita indet. & Indet. & Indet. \\
\hline
\end{tabular}

Tabla 2. Promedio ( $\pm \mathrm{DE}$ ) de los parámetros comunitarios de biodiversidad: riqueza de especies (S), cobertura vegetal (\%), índice de dominancia (D), índice de Simpson (1-D) e índice de Shannon-Wiener (H') para las geoformas estudiadas.

\begin{tabular}{lccc}
\hline $\begin{array}{l}\text { Parámetros } \\
\text { comunitarios }\end{array}$ & $\begin{array}{c}\text { Geoforma } \\
\text { Ladera Sur }\end{array}$ & $\begin{array}{c}\text { Geoforma } \\
\text { Depresión }\end{array}$ & $\begin{array}{c}\text { Geoforma } \\
\text { Ladera Norte }\end{array}$ \\
\hline (S) & $13,5 \pm 3,24^{\mathrm{b}}$ & $6 \pm 1,59^{\mathrm{a}}$ & $10,5 \pm 3,4^{\mathrm{b}}$ \\
(\%) & $88 \pm 5,89^{\mathrm{b}}$ & $75 \pm 16,68^{\mathrm{a}}$ & $86 \pm 13,06^{\mathrm{ab}}$ \\
(D) & $0,16 \pm 0,07^{\mathrm{a}}$ & $0,37 \pm 0,16^{\mathrm{b}}$ & $0,21 \pm 0,08^{\mathrm{a}}$ \\
$(1-\mathrm{D})$ & $0,84 \pm 0,07^{\mathrm{b}}$ & $0,63 \pm 0,16^{\mathrm{a}}$ & $0,79 \pm 0,08^{\mathrm{b}}$ \\
(H') & $2,09 \pm 0,35^{\mathrm{b}}$ & $1,23 \pm 0,37^{\mathrm{a}}$ & $1,81 \pm 0,36^{\mathrm{b}}$ \\
\hline
\end{tabular}

Letras minúsculas distintas representan diferencias significativas $(p<0,05)$.

$p \leq 0,0001)$. La Tabla 3 muestra el resultado de comparaciones pareadas por medio del análisisANOSIM, donde se aprecia las diferencias significativas en todos los casos $(p<0,05)$. Los resultados obtenidos con la aplicación del análisis
SIMPER para determinar la contribución de especies para cada geoforma se muestra en la Tabla 4. Diferentes especies de plantas son las que contribuyeron de manera más significativa tanto a la similitud dentro de cada grupo como 
Tabla 3. Valores de $\mathrm{p}<0,05$ de las comparaciones pareadas por ANOSIMentre la composición florística de las distintas geoformas estudiadas.

\begin{tabular}{lccc}
\hline & Geoforma Ladera Sur & $\begin{array}{c}\text { Geoforma } \\
\text { Depresión }\end{array}$ & $\begin{array}{c}\text { Geoforma Ladera } \\
\text { Norte }\end{array}$ \\
\hline Geoforma Ladera Sur & 0 & & \\
Geoforma Depresión & 0,0001 & 0 & 0 \\
Geoforma Ladera Norte & 0,0028 & 0,0001 & 0 \\
\hline
\end{tabular}

en la disimilaridad entre ellos, especialmente en la geoforma Depresión que corresponde a la vega. En este grupo, tres especies contribuyeron a la similitud dentro del mismo con más del $50 \%$ de la cobertura promedio; ellas son: Agrostis meyenii, Taraxacum officinale y Hordeum pubiflorum. En la geoforma Ladera Sur existió una mayor heterogeneidad taxonómica, aunque sólo una especie (Poa spiciformis) generó el valor más alto de la similitud dentro de este grupo con una cobertura promedio de $13 \%$. En cambio, en la geoforma Ladera Norte las especies que más contribuyeron a la similitud dentro de este grupo fueron: Taraxacum officinale, Acaena pinnatifida y Aira caryophyllea con una cobertura promedio de $42 \%$. Al encontrar diferencias significativas con ANOSIM, se identificaron los taxones que más contribuyeron a estas diferencias mediante el análisis SIMPER (Tabla 4).

\section{DISCUSIÓN}

Los resultados obtenidos indican que los componentes taxonómicos son diferentes en las distintas geoformas, especialmente en la Depresión, que corresponde a una vega, la cual se caracterizó por la presencia de una riqueza representada por 33 especies y con una cobertura promedio de $72 \%$, siendo la gramínea Agrostis meyenii la más importante en términos de cobertura, esto se ajusta a los resultados hallados en otros estudios en vegas realizados por Utrilla et al. (2008). Por otra parte, entre las geoformas del tipo ladera, la que presentó mayor riqueza fue la Ladera Sur con 34 especies, ésta se caracterizó por presentar una pendiente más suave y una mayor cobertura $(87,8 \%)$; a diferencia de la Ladera Norte, que presentó 27 especies y una cobertura de $79,7 \%$, la cual es utilizada como dormidero por las ovejas, encontrándose un alto porcentaje de heces.
Los índices de biodiversidad florística más altos correspondieron a las geoformas de Ladera Sur y Norte respectivamente, en comparación a la geoforma Depresión (Vega), donde se puede apreciar una presión antropogénica, debido a la intensidad del pastoreo. Esto ha causado la homogeneidad florística en términos de riqueza y cobertura, al existir tres especies dominantes, dos nativas (Agrostis meyenii y Hordeum pubiflorum) y una introducida (Taraxacum officinale); esto se aprecia en los resultados arrojados por los índices de Dominancia y Shannon, lo cual se ajusta a otros estudios realizados en vegas o mallines en Argentina, que evidencian el efecto del pastoreo (Boelcke et al. 1985; Oliva et al. 2001; Utrilla et al. 2006; Suárez et al. 2010; Bertani, 2011). Lo anterior estaría indicando que el sobrepastoreo genera efectos evidentes que se manifiestan en cambios en la vegetación, al sustituirse especies por otros taxones que son propios de sectores áridos. En este caso, la evidencia de este cambio es la presencia de la herbácea exótica Taraxacum officinale (diente de león), la cual es la segunda especie más importante en términos de cobertura en la vega (geoforma Depresión). Esta fue introducida intencionalmente en Magallanes el año 1882 (Moore \& Goodall, 1977). Se caracteriza por presentar valores nutricionales de proteína cruda de 15,1 a $16,28 \%$ y una digestibilidad aparente de la materia seca de 78,17 a 78,20\% (Domínguez et al. 2019). Estos valores no hacen a esta especie una excelente forrajera al compararla con un Trifolium repens (trébol blanco), que tiene $20,54 \%$ de proteína cruda y $73 \%$ de digestibilidad, pero se encuentra dentro de los rangos aceptables, considerando que se trata de una especie exótica invasora exitosa, la cual manifiesta una amplia tolerancia ambiental, debido a la presencia de mecanismos de evolución adaptativa de acuerdo a Molina-Montenegro et al. (2018), pudiéndose 
Tabla 4. Especies que contribuyen a diferenciar la composición florística de las geoformas estudiadas (Ladera Sur, Depresión y Ladera Norte) de acuerdo al análisis SIMPER. Las especies que contribuyen más en porcentaje a cada grupo están marcadas en gris.

\begin{tabular}{|c|c|c|c|c|}
\hline Especies & Geoforma Ladera Sur & Geoforma Depresión & $\begin{array}{c}\text { Geoforma Ladera } \\
\text { Norte }\end{array}$ & Origen \\
\hline Agrostis meyenii & 0 & 25,9 & 0 & \\
\hline Taraxacum officinale & 9 & 16,6 & 19,4 & Introducida \\
\hline Poa spiciformis & 13 & 0 & 7,5 & \\
\hline Hordeum pubiflorum & 2,22 & 11,1 & 1,22 & \\
\hline Acaena pinnatifida & 5 & 0 & 10,9 & \\
\hline Carex acaulis & 7,78 & 4,1 & 0,222 & \\
\hline Aira caryophyllea & 0 & 0,0769 & 11,7 & Introducida \\
\hline Poa pratensis & 5 & 3,54 & 3,22 & Introducida \\
\hline Acaena magellanica & 5,06 & 1,15 & 4,44 & \\
\hline Festuca gracillima & 8,33 & 0 & 1,11 & \\
\hline Eleocharis nubigena & 2,44 & 4,1 & 0 & \\
\hline Chiliotrichum diffusum & 5,89 & 0 & 0,889 & \\
\hline Capsella bursa-pastoris & 0 & 0,244 & 5,44 & Introducida \\
\hline Berberis microphylla & 2,78 & 0 & 2,56 & \\
\hline Syntrichia sp. & 4,28 & 0,385 & 1 & \\
\hline Hypochaeris incana & 3,33 & 0 & 0 & \\
\hline Cerastium arvense & 1,51 & 0,154 & 1,72 & Introducida \\
\hline Erodium cicutarium & 0 & 0 & 2,78 & Introducida \\
\hline Armeria maritima & 1,67 & 0,128 & 1,22 & \\
\hline Hypochaeris palustris & 0 & 1,46 & 0 & \\
\hline Luzula alopecurus & 1,61 & 0 & 0,389 & \\
\hline Olsynium biflorum & 0,944 & 0 & 0,889 & \\
\hline Festuca magellanica & 1,11 & 0,385 & 0,0556 & \\
\hline Colobanthus subulatus & 0,667 & 0,513 & 0 & \\
\hline Carex sorianoi & 1,11 & 0,256 & 0 & \\
\hline Arjona patagonica & 0,389 & 0 & 0,778 & \\
\hline Viola maculata & 0,333 & 0,0256 & 0,778 & \\
\hline Carex austroamericana & 1,11 & 0 & 0 & \\
\hline Symphyotrichum vahlii & 0,333 & 0,385 & 0 & \\
\hline Rumex acetosella & 0,556 & 0 & 0,389 & Introducida \\
\hline Oxalis loricata & 0,311 & 0 & 0,533 & \\
\hline Berberis empetrifolia & 0,556 & 0 & 0 & \\
\hline Acaena poeppigea & 0,556 & 0 & 0 & \\
\hline Phleum alpinum & 0 & 0,256 & 0 & \\
\hline
\end{tabular}


Tabla 4. continuación.

\begin{tabular}{|c|c|c|c|c|}
\hline Especies & $\begin{array}{c}\text { Cobertura promedio en la } \\
\text { geoforma Ladera Sur }\end{array}$ & $\begin{array}{l}\text { Cobertura promedio en } \\
\text { la geoforma Depresión }\end{array}$ & $\begin{array}{c}\text { Cobertura promedio } \\
\text { en la geoforma } \\
\text { Ladera Norte }\end{array}$ & Origen \\
\hline Cerastium fontanum & 0 & 0,256 & 0 & Introducida \\
\hline Arjona pusilla & 0 & 0,205 & 0 & \\
\hline Azorella monantha & 0,333 & 0 & 0 & \\
\hline Myosotis stricta & 0,0889 & 0,0128 & 0,222 & Introducida \\
\hline Lobelia oligophylla & 0 & 0,187 & 0 & \\
\hline Senecio miser & 0,333 & 0 & 0 & \\
\hline Juncus scheuchzerioides & 0 & 0,167 & 0 & \\
\hline Graminea indet. & 0 & 0,128 & 0 & \\
\hline Rumex crispus & 0 & 0,128 & 0 & Introducida \\
\hline Rytidosperma virescens & 0 & 0,128 & 0 & \\
\hline Adesmia lotoides & 0 & 0 & 0,167 & \\
\hline Leptinella scariosa & 0 & 0,103 & 0 & \\
\hline Calceolaria uniflora & 0 & 0 & 0,111 & \\
\hline Gentianella magellanica & 0 & 0,0538 & 0 & \\
\hline Draba verna & 0 & 0,0128 & 0,0556 & Introducida \\
\hline Festuca pyrogea & 0,0556 & 0 & 0 & \\
\hline Nassauvia aculeta & 0,0556 & 0 & 0 & \\
\hline Alopecurus magellanicus & 0 & 0,0256 & 0 & \\
\hline Peltigera sp. & 0,0333 & 0 & 0 & \\
\hline Geranium core-core & 0 & 0,0128 & 0 & \\
\hline Briófita indet. & 0 & 0,00769 & 0 & \\
\hline Contribución total & 87,8 & 72,2 & 79,7 & \\
\hline Nro. Total de Especies & 34 & 33 & 27 & \\
\hline
\end{tabular}

encontrar en todas las provincias y áreas protegidas de la región de Magallanes: Parque Nacional Torres del Paine, Parque Nacional Pali Aike, Monumento Natural Cueva del Milodón, Reserva Nacional Magallanes (Vidal et al. 2015; Domínguez et al. 2006; Domínguez, 2010; Dollenz, 1981), creciendo en forma espontánea en zonas áridas, humedales y especialmente en cultivos de alfalfa.

Por otra parte, la situación que muestran los indices de biodiversidad se relaciona al análisis de ordenamiento multidimensional (nMDS) basado en la presencia y su cobertura de especies, para evaluar la similitud o diferencias de la vegetación relacionada a las tres geoformas asociadas a la vega. A pesar de determinarse diferencias significativas $(p<0,05)$ entre la vegetación asociada a las geoformas, éstas muestran que la vega se ha ido empobreciendo, en términos de riqueza de especies y cobertura, compartiendo taxones que corresponden a condiciones más áridas entre las geoformas de laderas, lo cual coincide con estudios realizados por Soriano y Movia (1986), Sancholuz y Chaia (1993) y Pucheta et al. (1998), entre otros.

Lamentablemente no se conocen las condiciones iniciales, sin pastoreo, de las tres geoformas y especialmente de la vega; pero sí la presión del pastoreo continua sobre esta última. Estos cambios pueden llevar a una menor oferta en términos de calidad y en cantidad de forraje, 
debido a la sustitución por especies de ambientes más áridos, que puede tener un impacto directo en la ganadería, producto de la disminución de la calidad nutricional por el reemplazo de especies.

En este sentido, la actividad ganadera que consume el forraje producido sobre pastizales húmedos conocidos como vegas o mallines en Magallanes, deberá evaluar estrategias de manejo destinadas a mitigar los cambios en la composición y estructura en las vegas, e implementar planes de buenas prácticas para recuperar la cubierta vegetal y así mantener la biodiversidad y producción de forraje de calidad nutricional.

\section{CONCLUSIONES}

El pastoreo sobre geoformas asociadas a una vega sí genera diferencias en la composición y estructura florística de la vegetación. Los resultados obtenidos en este estudio permiten inferir que el pastoreo genera una homogeneidad florística en la geoforma Depresión (Vega) en términos de riqueza y cobertura de especies, al compararla con las otras geoformas. Además, se aprecia la sustitución de especies por otros taxones nativos e introducidos, que son propios de ambientes más áridos, entre los cuales destaca Taraxacum officinale. En este sentido, la actividad ganadera que usa el forraje producido por pastizales húmedos, conocidos como vegas o mallines en Magallanes, deberá evaluar estrategias de manejo destinadas a mitigar los cambios en la composición y estructura, junto con implementar planes de buenas prácticas para recuperar la biodiversidad y mantener una producción sustentable.

Por lo tanto, el pastoreo sin un manejo adecuado en sectores de vega puede tener un impacto negativo, en la ganadería, debido a la pérdida de biodiversidad, en este tipo de ambiente.

\section{AGRADECIMIENTOS}

La presente publicación se realizó como parte del proyecto FIC-2019: Evaluación del Balance Hídrico de las Vegas: Una estrategia para mejorar la gestión Hídrica a nivel predial y aumentar la resiliencia al Cambio Climático, financiado por el GORE Magallanes y ejecutado por el INIA Kampenaike. También se agradece a Salvador Reyes y a Claudio Pérez por su colaboración.

\section{LITERATURA CITADA}

Bertani, L. A. (2011). Evaluación geoecológica de los paisajes del Departamento Minas (Provincia de Neuquén), para el estudio de la degradación de la tierra. Universidad Nacional de La Plata. Tesis Doctoral.

Boelcke, O., Correa, M. N., Moore, D. M., \& Roig, F. A. (1985). Catálogo de las Plantas Vasculares. En O. Boelcke, D. M. Moore \& F. A. Roig (Eds.), Transecta Botánica de la Patagonia Austral (pp. 129-255). Buenos Aires: CONICET.

Bray, J. R., \& Curtis, J. T. (1957). An Ordination of the Upland Forest Communities of Southern Wisconsin. Ecological Monographs, 27, 325-349.

Buono, G., Oesterheld, M., Nakamatsu, V., \& Paruelo, J.M. (2010). Spatial and temporal variation of primary production of Patagonian wet meadows. Journal of Arid Environments, 74, 1257-1261.

Clarke, K. R. (1993). Non-parametric multivariate analyses of change in community structure. Australian Journal of Ecology, 18, 117143.

Clarke, K. R., \& Green, R. H. (1988). Statistical design and analysis for a 'biological effects' study. Marine Ecology Progress Series, 46, 213-226.

Clarke, K. R., Somerfeld, P. J., \& Chapman, M. G. (2006). On resemblance measures for ecological studies, including taxonomic dissimilarities and a zero-adjusted BrayCurtis coefficient for denuded assemblages. Journal of Experimental Marine Biology and Ecology, 330, 55-80.

Correa, M. N. (1969, 1971, 1978, 1984, 1985, 1988, 1999). Flora Patagónica I-VIII. Colección Científica INTA. Buenos Aires.

Dollenz, O. (1981). Catálogo de las plantas vasculares de la reserva forestal Magallanes. I. Lista preliminar correspondiente al área del cerro Mirador y río de las Minas. Anales Instituto de la Patagonia, 12, 205-212.

Domínguez, E. (2010). Aportes al conocimiento de 
la flora y vegetación del Monumento Natural Cueva del Milodón, Región de Magallanes (XII), Chile. Chloris Chilensis, Año $13 \mathrm{~N}^{\circ} 2$. URL: http://www.chlorischile.cl.

Domínguez, E., Elvebakk, A., Marticorena, C., \& Pauchard, A. (2006). Plantas introducidas en el Parque Nacional Torres del Paine, Chile. Gayana Botánica, 63, 131-141.

Domínguez, E., Pérez, C., Suárez, A., Lira, R., Ivelic-Sáez, J., \& de la Fuente, J. (2019). Manual para estimar la cantidad de forraje disponible en pastizales naturales: una herramienta necesaria para un uso racional de estos ecosistemas. Instituto de Investigaciones Agropecuarias. Centro Regional de Investigación Kampenaike. Punta Arenas, Chile. Boletín INIA, 401.

Gaitán, J. J., López, C., \& Bran, D. (2011). Vegetation composition and its relationship with the environment in mallines of north Patagonia, Argentina. Wetlands Ecology and Management, 19, 121-130.

Gaitán, J., Bran, D., Raffo, F., \& Ayesa, J. (2015). Evaluación y cartografía de mallines de las zonas de Loncopué y Chos Malal, provincia del Neuquén. Comunicación técnica, No. 131, área recursos naturales relevamiento integrado. Instituto Nacional de Tecnología Agropecuaria (INTA).

Gandullo, R., \& Schmid, P. (2001). Análisis ecológico de mallines del Parque Provincial Copahue, Neuquén, Argentina. Agro sur, 29(2), 83-99.

Grima, D., Vázquez, M. L., \& Diez, P. G. (2015). Composición florística de pequeñas áreas de mallines con distintas exposición y pendiente. Informes Científicos Técnicos - UNPA, 7(1), 144-161. https://doi. org/10.22305/ict-unpa.v7i1.118

Hammer, Ø., Harper, D. A. T., \& Ryan, P. D. (2001). PAST: Palaeontological statistics software package for education and data analysis. Palaeontologia Electronica, 4(1), 9 pp. http://palaeo-electronica. org/2001_1/past/issue1_01.htm

Härdle, W. K., \& Simar, L. (2012). Applied multivariate statistical analysis. New York, EE.UU.: Springer.

Houghton, R. A. (2007). Balancing the global carbon budget. Annual Review of Earth and Planetary Sciences, 35, 313-347.

Kruskal, J. B. (1964). Nonmetric multidimensional scaling. Psychometrika, 29, 1-27.

Lord, M., Jewett, D., Miller, J. R., Germanoski, D., \& Chambers, J. C. (2011). Hydrologic processes influencing meadow ecosystems. In J. C. Chambers, J. R, Miller (Eds.),Great Basin Riparian Ecosystems: ecology, management and restoration (pp. 44-67). Washington: Island Press.

Mazzoni, E., \& Rabassa, J. (2013). Types and internal hydro-geomorphologic variability of mallines (wet-meadows) of Patagonia: Emphasis on volcanic plateaus. Journal of South American Earth Sciences, 46, 70182.

Molina-Montenegro, M. A., del Pozo, A., \& Gianoli, E. (2018). Ecophysiological basis of the Jack-and-Master strategy: Taraxacum officinale (dandelion) as an example of a successful invader. Journal of Plant Ecology, 11(1), 147-157.

Moore, D., \& Goodall, R. N. (1977). La flora adventicia de Tierra del Fuego. Anales Instituto Patagonia, 8, 263-274.

Olff, H., \& Ritchie, M. E. (1998). Effects of herbivores on grassland plant diversity. Trends in Ecology and Evolution, 13, 261265.

Oliva, G., González, L., Rial, P., \& Livraghi, E. (2001). El ambiente en la Patagonia Austral. En P. Borrelli \& G. Oliva (Eds.), Ganadería ovina sustentable en la Patagonia Austral (pp. 19-82). Buenos Aires: Ediciones INTA. Ormaechea, S. G., Utrilla, V. R., Suárez, D. D., \& Peri, P. L. (2010). Evaluación objetiva de la condición de mallines de Santa Cruz. EEA INTA Santa Cruz. Producción Animal, 12, 47-52.

Pisano, E. (1977). Fitogeografía de FuegoPatagonia chilena. I.- Comunidades vegetales entre las latitudes $52^{\circ}$ y $56^{\circ} \mathrm{S}$. Anales Instituto Patagonia, 8, 121-250.

Pucheta, E., Cabido, M., Díaz, S., \& Funes, G. (1998). Floristic composition, biomass, and aboveground net plant production in grazed and protected sites in a mountain grassland of central Argentina. Acta Oecologica, 
19(2), 97-105.

Rodríguez, R., \& Marticorena, A. (Eds.). (2019). Catálogo de las plantas vasculares de Chile. Universidad de Concepción.

Sancholuz, L., \& Chaia, W. (1993). Evolución de la carga ganadera en las provincias patagónicas y su relación con la desertificación. Gaceta Agronómica, 13(75), 243-250.

Scopel, A. L., Cantero, J. C., \& Silva, M. P. (2017). Región Humedales Valliserranos. Subregión Arroyos y mallines de las sierras centrales. En Benzaquén, L., Blanco, D.E, Bo, R., Kandus, P., Lingua, G., Minotti, P. \& Quintana, R. (Eds.), Regiones de humedales de la Argentina (pp. 173-183). Buenos Aires: Ministerio de Ambiente y Desarrollo Sustentable, Fundación Humedales/ Wetlands International, Universidad Nacional de San Martín y Universidad de Buenos Aires.

Servicio Agrícola y Ganadero (2004). El pastizal de Magallanes. Guía uso, condición actual y propuesta de seguimiento para determinación de tendencia. Gobierno de Chile. XII Región de Magallanes y Antártica Chilena, Punta Arenas, Chile.

Soriano, A., \& Movia, C. (1986). Erosión y desertización en Patagonia. Interciencia, 11, 77-83.

Suárez, D., Ormaechea, S., Peri, P. L., \& Utrilla, V. (2010). Caracterización objetiva de la condición en mallines de Santa Cruz.
Informe técnico. EEA INTA Santa Cruz.

Utrilla, V. R., Brizuela, M. A., \& Cibils, A. F. (2006). Structural and nutritional heterogeneity of riparian vegetation in Patagonia (Argentina) in relation to seasonal grazing by sheep. Journal of Arid Environments, 67, 661670.

Utrilla, V., Ferrante, D., Peri, P., Kofalt, J.C., \& Humano, G. (2008). Efecto de la dinámica hídrica edáfica y ambiental sobre la productividad y calidad forrajera de mallines en la Patagonia Austral. EEA INTA Santa Cruz. Informe Técnico Final. 31 p.

Utrilla, V., Brizuela, M., \& Cibils, A. F. (2005). Riparian habitats (mallines) of Patagonia. A key grazing resource for sustainable sheep-farming operations. Outlook on Agriculture, 34(1), 55-59.

Vázquez, M., Diez, P., \& Grima, D. (2013). Relación suelo. Humedad y composición fisonómica florística del mallín La Carlota. Contribuciones Científicas GAEA, 25, 197-209.

Vidal, O. J., Aguayo, M., Niculcar, R., Bahamonde, N., Radic, S., San Martín, C., Kusch, A., Latorre, J., \& Félez, J. (2015). Plantas invasoras en el Parque Nacional Torres del Paine (Magallanes, Chile): Estado del arte, distribución post-fuego e implicancias en restauración ecológica. Anales del Instituto de la Patagonia, 43, 75-96. 